\title{
miR-486-3p Influences the Neurotoxicity of a-Synuclein by Targeting the SIRT2 Gene and the Polymorphisms at Target Sites Contributing to Parkinson's Disease
}

\author{
Yan Wanga,b YuJie Cai ${ }^{a}$ Haili Huang ${ }^{c}$ Xiongjin Chen ${ }^{a, d} \quad$ Xiaoyi Chen ${ }^{d}$ \\ Xiaoting Chen $^{d}$ Hui Mai ${ }^{a, d}$ Xiaohui Lia ${ }^{a, d}$ Jianghao Zhao ${ }^{a, e}$ Jingqi Yang ${ }^{a, d}$ \\ Weihao Fan ${ }^{a, d}$ Pei Tang ${ }^{a, d} \quad Y^{2}$ sen Chen ${ }^{d}$ Keshen Lia,f Lili Cui ${ }^{a}$ \\ a'Guangdong Key Laboratory of Age-Related Cardiac and Cerebral Diseases, Affiliated Hospital of \\ Guangdong Medical University, Guangdong, 'Key Laboratory of Biomedical Information Engineering \\ of Ministry of Education, School of Life Science and Technology, Xi an Jiaotong University, Shanxi, \\ Institute of Plastic Surgery, Affiliated Hospital of Guangdong Medical University, Guangdong, \\ IInstitutes of Neurology, Affiliated Hospital of Guangdong Medical University, Guangdong, \\ eDepartment of Neurology, Dongguan People's Hospital, Guangdong, fStroke Center, Neurology \\ \& Neurosurgery Division, Clinical Medicine Research Institute \& the First Affiliated Hospital, Jinan \\ University, Guangzhou, China
}

\section{Key Words}

Parkinson's disease $\cdot$ rs2241703 • Polymorphism • SIRT2 $・$ miR-486-3p • $\alpha$-synuclein

\begin{abstract}
Background/Aims: Increasing evidence suggests the important role of sirtuin 2 (SIRT2) in the pathology of Parkinson's disease (PD). However, the association between potential functional polymorphisms in the SIRT2 gene and PD still needs to be identified. Exploring the molecular mechanism underlying this potential association could also provide novel insights into the pathogenesis of this disorder. Methods: Bioinformatics analysis and screening were first performed to find potential microRNAs (miRNAs) that could target the SIRT2 gene, and molecular biology experiments were carried out to further identify the regulation between miRNA and SIRT2 and characterize the pivotal role of miRNA in PD models. Moreover, a clinical case-control study was performed with 304 PD patients and 312 healthy controls from the Chinese Han population to identify the possible association of single nucleotide polymorphisms (SNPs) within the miRNA binding sites of SIRT2 with the risk of PD. Results: Here, we demonstrate that miR-486-3p binds to the 3' UTR of SIRT2 and influences the translation of SIRT2. MiR-486-3p mimics can decrease the level of SIRT2 and reduce a-synuclein $(\alpha$-syn)-induced aggregation and toxicity, which may contribute to the progression of PD.

Y. Wang, Y. Cai and H. Huang contributed equally to this work.

\begin{tabular}{ll}
\hline Lili Cui, Keshen Li & Guangdong Key Laboratory of Age-Related Cardiac and Cerebral Diseases \\
and Yusen Chen & Affiliated Hospital of Guangdong Medical University, Zhanjiang, Guangdong (China) \\
& E-Mail cuilili@gdmu.edu.cn; likeshen1971@163.com; chenyusen925@163.com
\end{tabular}
\end{abstract}


Interestingly, we find that a SNP, rs2241703, may disrupt miR-486-3p binding sites in the $3^{\prime}$ UTR of SIRT2, subsequently influencing the translation of SIRT2. Through the clinical casecontrol study, we further verify that rs2241703 is associated with PD risk in the Chinese Han population. Conclusion: The present study confirms that the rs2241703 polymorphism in the SIRT2 gene is associated with PD in the Chinese Han population, provides the potential mechanism of the susceptibility locus in determining PD risk and reveals a potential target of miRNA for the treatment and prevention of PD.

(C) 2018 The Author(s)

Published by S. Karger AG, Basel

\section{Introduction}

Parkinson's disease (PD), one of the most common age-related neurodegenerative disorders worldwide, is pathologically characterized by the progressive loss of dopaminergic neurons within the substantia nigra pars compacta of the midbrain and the presence of Lewy bodies (LBs), mainly composed of accumulated misfolded $\alpha$-synuclein ( $\alpha$-syn) protein in surviving neurons $[1,2]$. Genetic and environmental risk factors have recently gained increasing attention as possible causes of PD. Significant efforts based on large-scale unbiased genome-wide association studies (GWAS) of PD patients have also successfully identified a number of loci that alter an individual's susceptibility to PD [3-8]. However, It is necessary to verify these susceptible loci and identify new susceptible loci in different populations, and translation of the genetic causes of this disease into a pathobiological understanding is largely lacking and remains controversial [9].

SIRT2, which is a NAD+-dependent deacylase, is widely involved in biological and disease processes, such as inflammation, metabolism, neurodegeneration and tumorigenesis [1015]. Sirtuin 2 (SIRT2) is the most abundant sirtuin in the brain and is closely associated with aging [16]. Increasing evidence has suggested an important role for SIRT2 in the pathology of PD in recent years. It has been reported that elevation of SIRT2 worsens motor impairment, while inhibition of SIRT2 diminishes striatal DA depletion and improves behavioral abnormalities in rotenone-treated rats [17]. Neurodegeneration induced by a chronic MPTP regimen is prevented by the genetic deletion of SIRT2 in mice [18]. Moreover, the inhibition of SIRT2 in cellular and Drosophila models of PD reduces $\alpha$-syn-mediated toxicity [19] and also alleviates neuropathology in several models of synucleinopathy by regulating levels of $\alpha$-syn acetylation, indicating the potential of SIRT2 as a therapeutic intervention for PD by targeting $\alpha$-syn $[20,21]$.

In the present study, we identified for the first time a novel single nucleotide polymorphism (SNP) in SIRT2 (rs2241703) associated with susceptibility to PD in the Chinese Han population. Moreover, we provided evidence that the SNP rs2241703 influences the binding efficiency between miR-486-3p and SIRT2, leading to changes in the expression levels of SIRT2, which in turn influence the neurotoxicity and aggregation of $\alpha$-syn, thus potentially contributing to the progression and risk of PD.

\section{Materials and Methods}

Cell culture

The HEK293T, SH-SY5Y and U87 cell lines were obtained from the Shanghai Cell Institute Country Cell Bank (Shanghai, China). All cell lines were grown in DMEM supplemented with $10 \%$ fetal bovine serum (FBS) (Gibco, USA), $100 \mathrm{U} / \mathrm{mL}$ penicillin G and $100 \mu \mathrm{g} / \mathrm{mL}$ streptomycin (Sigma, USA). The cells were maintained at $37^{\circ} \mathrm{C}$ in a humidified $5 \% \mathrm{CO}_{2}$ incubator.

Plasmid constructs

To generate luciferase reporter constructs containing the wild-type 3' UTR region of SIRT2, $397 \mathrm{bp}$ in the 3' UTR of SIRT2 was amplified and cloned into the pLUC luciferase vector using the restriction enzymes Xhol/BamHI (Promega, USA). Luciferase reporter constructs containing different rs2241703 genotypes 


\section{Cellular Physiology Cell Physiol Biochem 2018;51:2732-2745

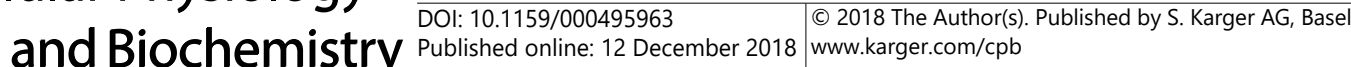

Wang et al.: The Mechanism of rs2241703 Associated with Risk of PD

were generated using the QuikChange ${ }^{\circledR}$ Site-Directed Mutagenesis Kit (Stratagene, USA) employing the wild-type 3' UTR of SIRT2 as the template. SIRT2 expression constructs were generated by subcloning the coding regions of SIRT2 into the GV141 vector using the restriction enzymes XhoI/KpnI. $\alpha$-syn expression constructs were generated by subcloning the coding region of $\alpha$-syn into the GV230 vector using the restriction enzymes XhoI/Kpn, resulting in the fusion of an EGFP tag to the C-terminus of $\alpha$-syn. The primers used are all listed in Table 1 . All constructs were verified via sequencing.

\section{RNA extraction and quantitative real-time reverse transcription PCR ( $q R T-P C R$ )}

Total RNA was extracted from peripheral blood leukocytes or cultured cells using TRIzol reagent (Invitrogen, USA) according to the manufacturer's instructions. The cDNA used to examine SIRT2 was synthesized with the PrimeScript ${ }^{\mathrm{TM}}$ RT reagent kit (TaKaRa, USA) according to the manufacturer's instructions. The cDNA employed to examine miR-486-3p was synthesized using the miRcute miRNA cDNA First-Strand Synthesis Kit (TIANGEN, China) according to the manufacturer's instructions. The expression of SIRT2 was examined using SYBR@ Premix Ex Taq ${ }^{\text {TM }}$ II (TaKaRa, USA), and GAPDH served as an internal reference. The expression of miR-486-3p was examined using the miRcute miRNA qPCR Detection Kit (TIANGEN, China), and U6 served as an internal reference. All experiments were performed in duplicate and repeated three times. The results are presented as the fold induction, determined using the $2^{-\Delta \Delta \mathrm{ct}}$ method. The primers for miR-486-3p and U6 were purchased from GenePharma, China.

\section{Western blot analysis}

Western blotting was performed according to standard western blotting procedures. Cells were lysed in RIPA buffer containing protease inhibitor cocktail (Sigma) and $1 \mathrm{mM}$ phenylmethyl sulfonyl fluoride (Sigma, USA). Lysates were centrifuged at $15,000 \mathrm{~g}$ for $30 \mathrm{~min}$ at $4^{\circ} \mathrm{C}$. Supernatants were collected, and protein concentrations were determined by the BCA Protein Assay Kit (Thermo, USA). Proteins were then separated via $12 \%$ SDS-PAGE and transferred to a polyvinylidene fluoride (PVDF) membrane (Millipore, USA). After blocking in 5\% nonfat milk, the membranes were incubated with the following primary antibodies: anti$\alpha$-tubulin (Abcam; 1:1, 000), anti-acetylated $\alpha$-tubulin (CST; 1:300), anti-SIRT2 (Abcam; 1:1, 000), anti- $\alpha$ synuclein (CST; $1: 1,000)$, and anti-GAPDH (CST; 1:1, 000). The proteins were visualized with enhanced chemiluminescence reagents (Pierce, USA).

Table 1. Sequences of primers used for experiments in this study

\begin{tabular}{|c|c|}
\hline Sequence Type & Primer Sequence \\
\hline rs2241703 & $\begin{array}{l}\text { 5'-TTTGGGAGCTGAAGGCAGAGAC-3' } \\
\text { 5'-AGGCATCTCTACCAGCCCCACT-3' }\end{array}$ \\
\hline \multicolumn{2}{|c|}{ Sequences of primers used for pLUC-SIRT2-WT construction } \\
\hline Sense & 5'-CACAACTCGAGTCCCCGCCACCTGCCAAGGACGA-3' \\
\hline Antisense & 5'-AAGGATCCCCCGAAGCTCCCTGTCCTGGAAG-3' \\
\hline \multicolumn{2}{|c|}{ Sequences of primers used for pluc-SIRT2-mutant construction } \\
\hline Sense & 5'- ATCTCTAACTGGCCCCACGGGGCCA-3' \\
\hline Antisense & 5'- CGTGGGGCCAGTTAGAGATGAGGGAG-3' \\
\hline \multicolumn{2}{|c|}{ Sequences of primers used for pluc-SIRT2-7bp delete construction } \\
\hline Sense & 5'- TAACCTCCCTCATCTCTAACACGGGGCCAGGGCTACCCCAG-3' \\
\hline Antisense & 5' - CCCCGTGTTAGAGATGAGGGAGGTTACTCCTTAGCCCAGG-3' \\
\hline \multicolumn{2}{|c|}{ Sequences of primers used for syn expression construction } \\
\hline Sense & 5'-TACCGGACTCAGATCTCGAGCGCCACCATGGATGTATTCATGAAAGGAC-3' \\
\hline Antisense & 5'-GATCCCGGGCCCGCGGTACCGTGGCTTCAGGTTCGTAGTCTTG-3' \\
\hline \multicolumn{2}{|c|}{ Sequences of primers used for SIRT2 expression construction } \\
\hline Sense & 5'-ACGGGCCCTCTAGACTCGAGCGCCACCATGGCAGAGCCAGACCCCTCTC-3' \\
\hline Antisense & 5'-AGTCACTTAAGCTTGGTACCGACTGGGGTTTCTCCCTCTCTGTTG-3' \\
\hline \multicolumn{2}{|c|}{ Sequences of primers used for GAPDH qRT-PCR (human) } \\
\hline Sense & 5'-AСССАСТССТССАССТTTGA-3' \\
\hline Antisense & 5'-CTGTTGCTGTAGCCAAATTCGT-3' \\
\hline \multicolumn{2}{|c|}{ Sequences of primers used for SIRT 2 qRT-PCR(human) } \\
\hline Sense & 5'-CACGCAGAACATAGATACCCTG-3' \\
\hline Antisense & 5'-CAGTGTGATGTGTAGAAGGTGC-3' \\
\hline \multicolumn{2}{|c|}{ Sequences of primers used for U6 qRT-PCR } \\
\hline Sense & 5'-ATTGGAACGATACAGAGAAGATT-3' \\
\hline Antisense & 5'-GGAACGCTTCACGAATTTG-3' \\
\hline \multicolumn{2}{|c|}{ Sequences of primers used for miR-486-3p qRT-PCR } \\
\hline Sense & 5'-ATAAATCGGGGCAGCTCAGTA-3' \\
\hline Antisense & 5'-TATGCTTGTTCTCGTCTCTGTGTC-3' \\
\hline
\end{tabular}




\section{Cellular Physiology Cell Physiol Biochem 2018;51:2732-2745

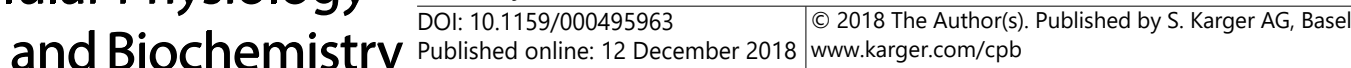

Wang et al.: The Mechanism of rs2241703 Associated with Risk of PD

\section{Luciferase reporter assays}

HEK293T cells were seeded into 96 -well plates at $2 \times 10^{4}$ cells per well one day before transfection, after which $200 \mathrm{ng}$ of the luciferase reporter construct (pLUC-SIRT2-WT or pLUC-SIRT2-mutant) and 450 ng of pLMP-miR-486-3p or the pLMP vector were cotransfected into HEK293 cells with Lipofectamine 2000. Forty-eight hours later, firefly and renilla luciferase activities were measured with a Dual-Luciferase Reporter System (Promega, USA) according to the manufacturer's instructions. Firefly luciferase activity was detected and used to normalize renilla luciferase activity.

\section{Evaluation of $\alpha$-synuclein aggregation}

To assess the effects of miR-486-3p on $\alpha$-syn aggregation, we cotransfected SH-SY5Y and U87 cells with $\alpha$-syn and synphilin-1 (an established paradigm that leads to inclusion formation in SY5Y and U87 cells), with or without miR-486-3p. After $48 \mathrm{~h}$, the cells were fixed for $15 \mathrm{~min}$ with $4 \%$ formaldehyde in PBS, followed by permeabilization with $0.2 \%$ Triton X-100 for $10 \mathrm{~min}$. Cells were incubated with DAPI for $1 \mathrm{~min}$ at room temperature and subsequently photographed by confocal microscope (TCS SP5 II, Leica).

\section{Triton insolubility assay}

For total protein extraction, cells were lysed in NP-40 buffer (Beyotime, China) supplemented with a protease inhibitor cocktail tablet (Sigma, USA). The samples were sonicated 3 times for $30 \mathrm{sec}$, with a 1-min incubation on ice between each sonication step. Proteins were separated from debris via centrifugation at $18,000 \mathrm{~g}$ for $30 \mathrm{~min}$ at $4^{\circ} \mathrm{C}$. Total protein $(1 \mathrm{mg}$ ) from cells transiently expressing $\alpha$-syn and synphilin-1, with or without miR-486-3p, for $48 \mathrm{~h}$ was incubated with $1 \%$ Triton X-100 on ice for $30 \mathrm{~min}$. The protein fractions were separated via centrifugation at $15,000 \mathrm{~g}$ for $60 \mathrm{~min}$ at $4^{\circ} \mathrm{C}$. The soluble protein fraction was subsequently collected, and the insoluble protein fraction pellet was resuspended in $40 \mu \mathrm{L}$ of $2 \%$ SDS Tris$\mathrm{HCl}$ buffer, $\mathrm{pH}$ 7.4, followed by sonication for 10s. The total protein and T-Insoluble fractions were loaded and resolved via SDS-PAGE and immunoblotted as described above.

\section{Immunocytochemistry}

Cells were fixed in 4\% paraformaldehyde in PBS for 15 min, washed with PBS three times, and permeabilized with $0.2 \%$ Triton X-100 in PBS for 10 min. After washing with PBS again and blocking with $1 \%$ goat serum for $30 \mathrm{~min}$, cells were incubated with anti-tyrosine hydroxylase antibody (abcam; 1:200) diluted in $1 \%$ goat serum at room temperature for $1 \mathrm{~h}$ and washed with PBS and incubated with Cy3conjugated goat anti-rabbit IgG (EarthOx; 1:800) diluted in 1\% goat serum for $1 \mathrm{~h}$ at room temperature. For nuclear staining, cells were incubated with DAPI for $1 \mathrm{~min}$ at room temperature. Cells were washed five times with PBS and analyzed under a fluorescence microscope (EVOS fl auto, life, USA).

\section{MTT assay}

Cell viability was analyzed in vitro with the tetrazolium salt 3-(4, 5-dimethylthiazol-2-yl)-2, 5-diphenyltetrazolium bromide (MTT) reagent. Briefly, 2, 000 cells from each group were plated in each well of a 96-well plate in $200 \mu \mathrm{L}$ of medium. To analyze cell viability, $20 \mu \mathrm{L}$ of MTT substrate at a concentration of $5 \mathrm{mg} / \mathrm{mL}$ in PBS was added to each well. The plates were then returned to a standard tissue incubator for an additional $4 \mathrm{~h}$. The medium was subsequently removed, and the cells were solubilized in $150 \mu \mathrm{L}$ of dimethylsulfoxide (DMSO), shaken for $10 \mathrm{~min}$ and subjected to colorimetric analysis (wavelength: $490 \mathrm{~nm}$ ).

\section{Subjects}

In total, 304 PD patients (aged 45-91; 180 men and 124 women) and 312 healthy normal volunteers (aged 41-90; 170 men and 142 women) were recruited in this case-control study. All PD subjects were enrolled in the Department of Neurology of the Affiliated Hospital of Guangdong Medical University (Zhanjiang, China) between August 2006 and August 2017. All patients were examined, and their diagnosis was confirmed by expert neurologists. The diagnostic criteria for PD were based on the diagnostic criteria developed by the National Medical Journal of China from August 2006 to October 2015 and the MDS clinical diagnostic criteria for PD from October 2015 to August 2017. All participants came from the Chinese Han population of Guangdong Province, China. Patients who had a history of or suffered from cerebrovascular disease, brain trauma, intracranial infection, other known neurological degenerative diseases, parkinsonism plus syndromes and Parkinson's syndrome caused by drugs and/or chemical toxicity were excluded from 
the present study. The control subjects were individuals who were free of PD, related neurologic disorders and other cardiovascular diseases. The demographic data and clinical features of the participants included as PD patients and healthy controls are shown in Table 2. Moreover, the age at onset of PD and the time until diagnosis were recorded for the $221 \mathrm{PD}$ patients in the PD group.

\section{Genotyping}

A fasting venous blood sample of $2 \mathrm{~mL}$ was extracted from each subject at the elbow on the morning after diagnosis and was treated with ethylenediamine tetraacetic acid dipotassium salt (EDTA-K2) to prevent coagulation. Within 1-4 h, $0.5 \mathrm{~mL}$ of the blood sample was added to two 1.5-mL EP tubes. Genomic DNA was isolated from peripheral blood samples using a blood Genomic DNA Extraction Kit (TIANGEN, China). The DNA was genotyped using the ABI PRISM SNapShot method (Applied Biosystems). Briefly, the SNapShot reaction was performed in a 10-mL final volume containing SNapShot Multiplex Ready Mix (5 mL), primer mix (0.02-0.6 mmol/L), and template ( $4 \mathrm{~mL}$ ) consisting of the multiplex PCR products, which were purified with the QIAquick PCR Purification Kit (QIAGEN, China). The cycling program included 25 cycles of $94^{\circ} \mathrm{C}$ for 30 seconds, $57^{\circ} \mathrm{C}$ for 30 seconds, and $72^{\circ} \mathrm{C}$ for 40 seconds. The extension products were purified via incubation with $1 \mathrm{U}$ of shrimp alkaline phosphatase (Promega, USA) for 15 minutes at $37^{\circ} \mathrm{C}$ and subsequent incubation at $80^{\circ} \mathrm{C}$ for 15 minutes to inactivate the enzyme. The purified products $(0.5 \mathrm{~mL})$ were mixed with $9 \mathrm{~mL}$ of formamide and $0.5 \mathrm{~mL}$ of the GeneScan-120 LIZ Size Standard (Applied Biosystems, USA) and separated via capillary electrophoresis (ABI PRISM310 Genetic Analyzer; Applied Biosystems, USA). The results were analyzed with GeneMapper 3.0 software (Applied Biosystems, USA). For quality control, random duplicate samples (5\%) were run for each sequence analysis.

\section{Statistical analysis}

Genotype and allele frequencies were estimated by counting. Hardy-Weinberg equilibrium between the expected and observed genotype distributions was assessed using the Chi-square test. Allele and genotype distributions were also compared using the Chi-square test. Associations were expressed as odds ratios (OR) or risk estimates with $95 \%$ confidence intervals (CIs). The nonparametric Mann-Whitney test was employed to compare SIRT2 expression between the genotype groups. Other data were analyzed with GraphPad Prism 6 software. Results are presented as the mean \pm s.e.m.; the means between two groups were compared with an unpaired, two-tailed Student's t test. The means among more than two groups were determined using one-way ANOVA. $P<0.05$ was considered statistically significant.

\section{Results}

SIRT2 is the target of miR-486-3p, and the SNP rs2241703 influences the binding efficiency Bioinformatics analysis (DIANA, Targetscan, PITA) was first performed to predict and analyze potential miRNAs targeting the 3' UTR of SIRT2, thenmiR-486-3p was screened as the candidate for targeting the SIRT2 gene (data not shown). Moreover, we found that the predicted miR-486-3p binding site within the 3' UTR of the SIRT2 gene included a SNP site, rs2241703. Subsequently, a luciferase reporter assay was performed to verify whether miR486-3p binds to the 3' UTR of SIRT2 and whether the rs2241703 SNP could influence this interaction (Fig. 1A). As shown in Fig. 1B, there was significantly reduced binding between miR-486-3p and the A allele relative to the $\mathrm{G}$ allele based on the reduced luciferase activity in the presence of the G allele, suggesting that miR-486-3p directly binding to the 3' UTR of the SIRT2 gene and the rs2241703 A allele alters the binding efficiency between them (Fig. 1B).

We next investigated the effect of miR-486-3p on the endogenous expression of SIRT2 in HEK293 cells. As shown in Fig. 1C and D, when miR-486-3p was overexpressed or silenced, 
SIRT2 mRNA expression was in turn reduced or elevated, respectively, and the protein level of SIRT2 was also negatively correlated with the miR486-3p level (Fig. 1E and F), suggesting that miR-486$3 p$ negatively regulates the expression of SIRT2.

\section{The rs2241703}

poly morphis $\mathrm{m}$ influences the expression level of SIRT2 Then we further evaluated the expression of SIRT2 in peripheral blood mononuclear cells (PBMCs) from $P D$ patients and healthy controls with different genotypes to explore the possible associations between the SNPs and SIRT2 expression levels. As shown in Fig. 2A, the mRNA expression of SIRT2 was significantly higher in the PBMCs of PD patients than in those of healthy controls $(P=0.0234$ and $P=0.0151$ ), and the levels of miR-486-3p were lower in $\mathrm{PD}$ patients than in healthy controls (Fig. 2B). Then, we subdivided the two groups by genotype. Remarkably, the mRNA expression of SIRT2 in PD patients with the GG genotype was lower than in patients with the GA and AA genotypes, while no significant difference in the expression of SIRT2 was observed between healthy controls with the GG genotype and those with the GA or AA genotype (Fig. 2C and D). However, for miR-486-3p, there was no significant difference among the groups (Fig. 2C and D). This result provides the support that the presence of the A allele may influence the expression of SIRT2 and increase the risk of PD.

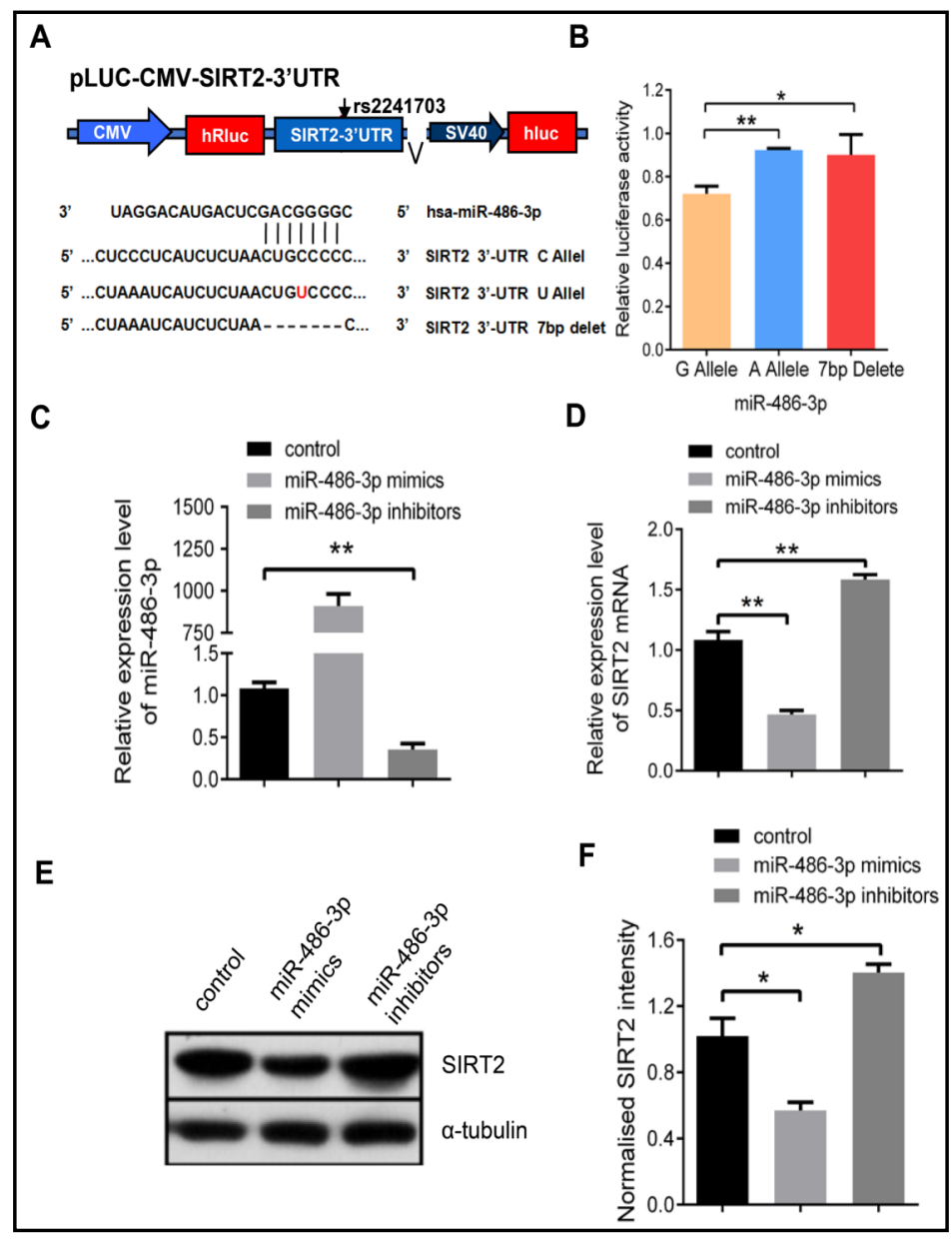

Fig. 1. SIRT2 is the target of miR-486-3p, and the SNP rs 2241703 influences their binding efficiency. (A) Schematic of the SIRT2 3' UTRluciferase constructs containing rs2241703 alleles and the predicted binding site for miR-486-3p in gene SIRT2; (B) relative reporter gene activity from constructs bearing the 3' UTR of SIRT2 with the rs2241703 G or rs2241703 A allele in HEK 293 cells. Luciferase activity was normalized to renilla activity; (C) levels of miR-486-3p after transfection of miR-486-3p mimics, antagomirs or NC in SH-SY5Y cells determined by qRT-PCR, relative to U6 as the control group; (D) The mRNA levels of SIRT2 after the transfection of miR-486-3p mimics, antagomirs or NC in SH-SY5Y cells determined by qRT-PCR, relative to GAPDH as the control group; (E, F) protein levels of SIRT2 after the transfection of miR-486-3p mimics, antagomirs or NC in SH-SY5Y cells were assessed by WB and and thegrey intensity analysis, relative to $\beta$-tubulin as the control group. Results are shown as the mean \pm s.e.m. from three experiments, ${ }^{* *} \mathrm{P}<0.01$. Differences between groups were analyzed using two-sided t tests. 
Fig. 2. The distribution of rs2241703 in miR-486-3P and SIRT2 expression levels in PD patients. (A) levels of SIRT2 in PBMCs of PD patients $(n=68)$ and healthy controls $(n=31)$ determined by qRT-PCR, relative to GAPDH levels; (B) levels of miR-486-3p in PBMCs of PD patients $(n=38)$ and healthy controls $(n=27)$ determined by qRT-PCR, relative to U6 levels; (C) levels of SIRT2 in PBMCs of PD patients with the GG $(n=48)$ and $G A+A A$ $(n=20)$ genotype and healthy controls with the GG $(n=20)$ and $\mathrm{GA}+\mathrm{AA}(\mathrm{n}=11)$ genotypes at rs2241703 determined by qRT-PCR, relative to GAPDH levels; (D) levels of SIRT2 and miR-486-3p in PBMCs of PD patients with the GG $(n=30)$ and $\mathrm{GA}+\mathrm{AA}(\mathrm{n}=8)$ genotype and healthy controls with the $\mathrm{GG}(\mathrm{n}=21)$ and $\mathrm{GA}+\mathrm{AA}(\mathrm{n}=6)$ genotype at rs2241703 determined by qRT-PCR, relative to U6 levels. Results are shown as the mean \pm s.e.m. from three experiments. All P-values are from two-sided t tests.

\section{MiR-486-3p reduces $\alpha$-syn inclusions and suppresses $\alpha$-Syn toxicity}

A recent study showed that SIRT2 governs $\alpha$-syn aggregation [21]. To confirm whether miR-486-3p regulates $\alpha$-syn aggregation through SIRT2, we established a $\alpha$-syn aggregation model in both SH-SY5Y and U87 cells, involving the co-expression of a C-terminally modified form of $\alpha$-syn with an increased propensity for aggregation and synphilin-1 (an $\alpha$-syn interactor that potentiates its aggregation) in neural cells [3]. With the transfection of miR-486-3p mimics, the formation of $\alpha$-syn inclusions significantly reduced, and the diffuse distribution of $\alpha$-syn was restored in SH-SY5Y cells (Fig. 3A) and U87 cells (Fig. 3B). Moreover, elevated levels of miR-486-3p also decreased insoluble $\alpha$-syn aggregation, corresponding to the above results (Fig. 3C). Notably, $\alpha$-tubulin is an important substrate related to SIRT2 and has been reported to contribute to the neurotoxicity of LBs [22]. Thus, we further measured the acetylation status of $\alpha$-tubulin in SH-SY5Y cells treated with miR486-3p mimics. As expected, the overexpression of miR-486-3p significantly increased the acetylation level of $\alpha$-tubulin, and this effect could be rescued by the overexpression of SIRT2 (Fig. 3D). The above findings suggest that miR-486-3p mimics increase the acetylation level of $\alpha$-tubulin by reducing the expression of SIRT2.Finally, we evaluated whether miR-486$3 p$ could contribute to $\alpha$-syn-induced neuronal injury. We established $\alpha$-syn-overexpressing and MPP+-induced SH-SY5Y cells as a PD cell model. As shown in Fig. 3E, concomitant with the decrease in $\alpha$-syn aggregation, miR-486-3p decreased the cytotoxicity of $\alpha$-syn according to an MTT assay. Accordingly, miR-486-3p also significantly increased the number of THpositive cells in $\alpha$-syn-overexpressing SH-SY5Y cells (Fig. 3F), suggesting that elevated miR486-3p levels rescued PD-like neuronal injury. 


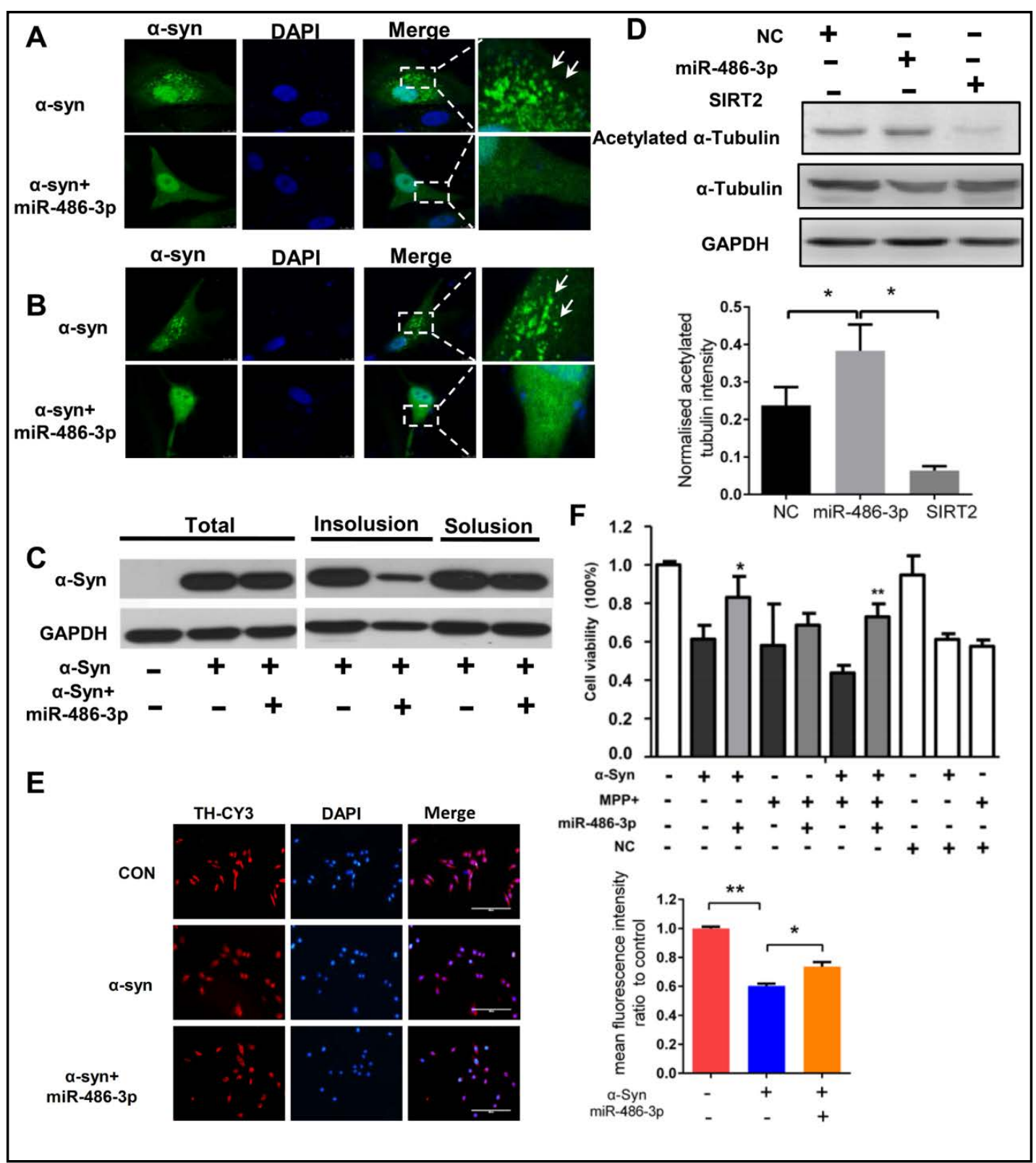

Fig. 3. MiR-486-3p reduces $\alpha$-Syn inclusions and suppresses $\alpha$-Syn toxicity. (A) SH-SY5Y cells transiently expressing $\alpha$-syn (green) and synphilin were co-transfected with NC and miR-486-3p, respectively, for 48 $\mathrm{h}$, and cells were processed for immunocytochemistry; (B) U87 cells transiently expressing $\alpha$-syn (green) and synphilin were co-transfected with NC or miR-486-3p, respectively, for $48 \mathrm{~h}$, and cells were processed for immunocytochemistry; (C) Triton X-100-insoluble and total cell fractions were assayed by WB, using GAPDH as a reference; (D) SIRT2, $\alpha$-tubulin and acetylated $\alpha$-tubulin were assessed by WB when cells were transfected with NC, miR-486-3p mimics and pcDNA-SIRT2, respectively, relative to GAPDH as the control group; (E) Cell were transfected with $\alpha$-syn or co-transfected with $\alpha$-syn and miR-486-3p mimics and immunostained for TH (red); nuclei are counterstained with DAPI (blue); (F) SH-SY5Y cells were treated with or without $\alpha$-syn, miR-486-3p or MPP+ and then were assayed by MTT; the results obtained with each treatment were normalized to $100 \%$. Results are shown as the mean \pm s.e.m; all P-values are from two-sided t tests. ${ }^{*} \mathrm{P}<0.05,{ }^{* *} \mathrm{P}<0.01$. 
Fig. 4. Schematic illustration of the mechanism by which the rs2241703 SNP contributes to the pathological process or risk of PD. MiR-486-3p binds to the 3' UTR of SIRT2 within the rs2241703 site, and the risk allele for rs2241703 disrupts a binding site for miRNA-486-3p and then increases the translation of SIRT2. Higher levels of SIRT2 could increase $\alpha$-syn-induced aggregation and toxicity and finally may contribute to the progression and risk of PD.

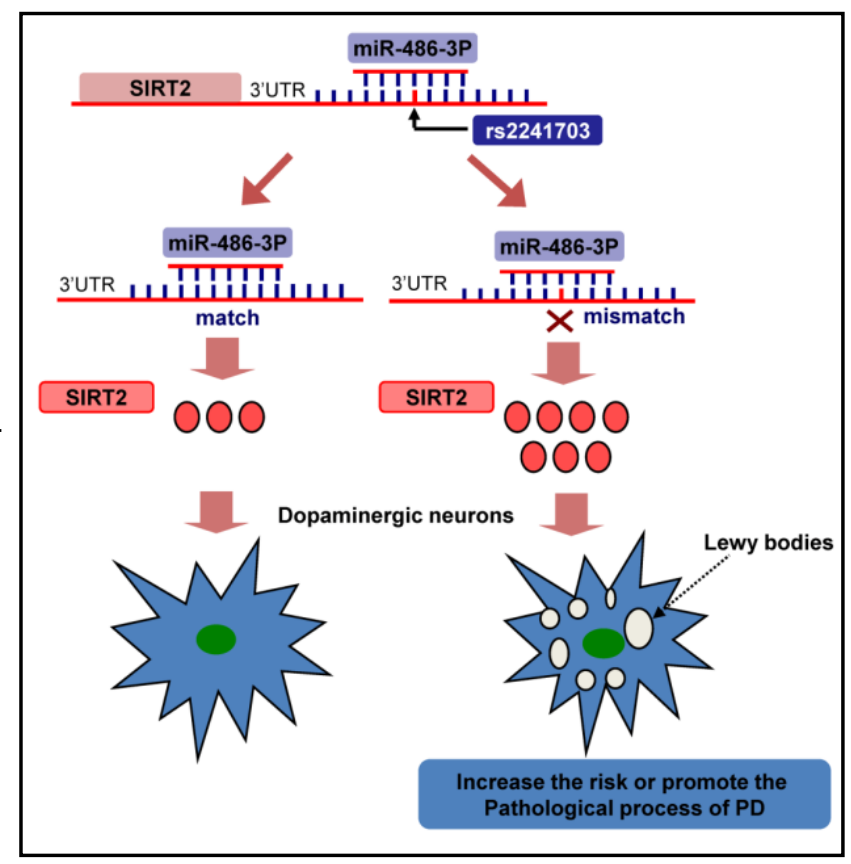

Demographic characteristics of PD patients and healthy controls

Based on the above results from the mechanism study, a clinical case-control study was preformed to further explore the possible association between rs2241703 and the risk of PD. A total of 304 patients diagnosed with PD and 312 unrelated genderand age-matched controls were recruited in the present study. The demographic and clinical characteristics of the study subjects are summarized in Table 2 . The mean age of the PD patients was $64.8 \pm 10.0$ years, while that of the control subjects was $62.3 \pm 10.0$ years. The gender (male/female) ratio was 1:0.69 in the case group and 1:0.83 in the control group. The age and gender distributions did not differ significantly between the PD patients and the controls $(P=0.236$ and 0.4298 , respectively). Furthermore, the onset and the duration time of PD were recorded for 222 PD patients, with average values of $64.5 \pm 10.4$ years and $63.8 \pm 3.90$ years, respectively.
Table 3. Distribution of the rs2241703 polymorphisms of the SIRT2 gene between PD patients and controls

\begin{tabular}{|c|c|c|c|c|}
\hline $\mathrm{rs} 2241703 \mathrm{G}>\mathrm{A}$ & Controls (312) & PD (304) & OR $(95 \% \mathrm{CI})$ & P-value \\
\hline GG & 240 & 212 & 1.00 (reference) & - \\
\hline GA & 68 & 86 & $0.991-2.068$ & 0.0553 \\
\hline $\mathrm{AA}$ & 4 & 6 & $0.473-6.101$ & 0.4119 \\
\hline $\mathrm{GG}+\mathrm{GA}$ & 244 & 218 & $0.981-2.043$ & 0.0769 \\
\hline $\mathrm{AA}+\mathrm{GA}$ & 72 & 92 & $1.010-2.073$ & 0.0454 \\
\hline $\mathrm{G}$ alleles & 548 & 510 & 1.00 (reference) & - \\
\hline A alleles & 76 & 98 & $1.003-1.914$ & 0.0472 \\
\hline$\geq 60$ years & Controls (134) & PD (176) & OR $(95 \% \mathrm{CI})$ & P-value \\
\hline GG & 104 & 116 & 1.00 (reference) & - \\
\hline GA & 28 & 57 & $1.081-3.083$ & 0.0235 \\
\hline $\mathrm{AA}$ & 2 & 3 & $0.2203-8.210$ & 0.7473 \\
\hline $\mathrm{GG}+\mathrm{GA}$ & 132 & 175 & $0.1863-6.871$ & 0.8932 \\
\hline $\mathrm{AA}+\mathrm{GA}$ & 30 & 60 & $1.074-2.992$ & 0.0245 \\
\hline G alleles & 236 & 289 & 1.00 (reference) & - \\
\hline A alleles & 32 & 63 & $1.016-2.544$ & 0.0413 \\
\hline Male & Controls (170) & $\mathrm{PD}(180)$ & OR $(95 \% \mathrm{CI})$ & P-value \\
\hline GG & 131 & 129 & 1.00 (reference) & \\
\hline GA & 37 & 49 & "0.8228 to $2.198 "$ & 0.2364 \\
\hline $\mathrm{AA}$ & 2 & 4 & "0.3655 to $11.29 "$ & 0.4089 \\
\hline $\mathrm{GG}+\mathrm{GA}$ & 168 & 178 & "0.3411 to $10.45 "$ & 0.4595 \\
\hline $\mathrm{AA}+\mathrm{GA}$ & 39 & 53 & "0.8541 to $2.230 "$ & 0.1873 \\
\hline G alleles & 299 & 307 & 1.00 (reference) & \\
\hline A alleles & 41 & 57 & "0.8791 to $2.085 "$ & 0.1679 \\
\hline Female & Controls (142) & $\mathrm{PD}(124)$ & OR $(95 \% \mathrm{CI})$ & P-value \\
\hline GG & 109 & 83 & 1.00 (reference) & \\
\hline GA & 31 & 39 & "0.9518 to $2.868 "$ & 0.0730 \\
\hline $\mathrm{AA}$ & 2 & 2 & "0.1811 to $9.522 "$ & 0.7868 \\
\hline $\mathrm{GG}+\mathrm{GA}$ & 140 & 122 & "0.1592 to $8.273 "$ & 0.8913 \\
\hline $\mathrm{AA}+\mathrm{GA}$ & 33 & 41 & "0.9507 to $2.800 "$ & 0.0744 \\
\hline G alleles & 249 & 205 & 1.00 (reference) & \\
\hline A alleles & 35 & 43 & "0.9205 to $2.419 "$ & 0.1028 \\
\hline
\end{tabular}




\section{Cellular Physiology Cell Physiol Biochem 2018;51:2732-2745

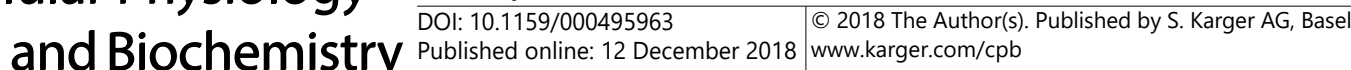

Wang et al.: The Mechanism of rs2241703 Associated with Risk of PD

Association of SIRT2 polymorphisms between PD cases and controls

All enrolled samples were successfully genotyped for the rs2241703 SNP, and no deviations in Hardy-Weinberg equilibrium were observed for the rs2241703 polymorphisms in the PD and control subjects (data not shown). The allele and genotype frequencies of the SIRT2 polymorphism in the entire study population are shown in Table 3. Significant differences in the allele frequencies of the rs2241703 SNP and the dominant model between the PD cases and controls were observed (allele $P=0.0472$, dominant model $P=0.0454$ ), with $\mathrm{AA}+\mathrm{GA}$ genotype or A allele carriers presenting a higher risk of PD. Moreover, as shown in Table 3, the AA+GA genotype and A allele frequencies of rs2241703 in the late-onset PD (LOPD) subgroup ( $>60$ years) differed significantly from those in the corresponding controls ( $P=0.0245$ for the dominant model and $P=0.0413$ for the allele). However, no statistically significant differences were observed between case and control subjects of different genders (Table 3), suggesting that gender is not a risk factor contributing to PD susceptibility associated with this SNP locus. In conclusion, our case-control results revealed that the rs2241703 polymorphism, located in the SIRT2 3' UTR, is significantly associated with susceptibility to PD in the Chinese Han population.

\section{Discussion}

In the present study, we reported that miR-486-3p targets SIRT2 and identified a SNP, rs2241703, within the binding site of the 3' UTR of SIRT2 that was significantly associated with PD in the Chinese Han population. Moreover, mechanically, this SNP influenced the expression level of SIRT2 by disrupting the miR-486-3p binding sites in the 3' UTR of SIRT2, and miR-486-3p influenced SITR2 levels and the aggregation and neurotoxicity of $\alpha$-syn, thereby contributing to the progression and risk of PD (Fig. 4).

It has been reported that SIRT2, as a strong deacetylase [23-26], is highly expressed in the brain and is markedly associated with aging [27, 28]; additionally, SIRT2 was evaluated in the brains of PD patients, suggesting a potential role in PD development [29]. It has been confirmed that selective inhibitors of SIRT2 can protect against $\alpha$-syn-mediated toxicity and dopaminergic cell death, both in vitro and in a Drosophila model of PD [19]. Notably, a recent study showed that $\alpha$-syn can be deacetylated by SIRT2, and $\alpha$-syn acetylation is a key regulatory mechanism governing aggregation and toxicity, demonstrating the potential therapeutic value of SIRT2 inhibition in synucleinopathies [21]. The present study confirmed that mimics of miR-486-3p, which acts as an inhibitor of SIRT2, could reduce the aggregation state of LBs and $\alpha$-syn-induced neurotoxicity. The results provide evidence of the mechanism by which the rs 2240713 locus affects PD susceptibility and reveal a potential target of miRNA for the treatment and prevention of PD.

Increasing evidence suggests that dysregulated miRNAs directly contribute to the pathogenesis of a variety of human diseases [30-34]. SNPs in miRNA target sites in the 3' UTR of mRNAs are referred to as polymorphisms in microRNAs and their target sites (polymiRTSs) and can affect mRNA half-life, resulting in decreased protein levels due to mRNAmiRNA interactions and increased susceptibility to many diseases [30-33, 35, 36], including PD [33]. Two SNPs located in the 3' UTR of human $\alpha$-syn, rs17016074 and rs10024743, can influence the miRNA-mediated repression of $\alpha$-syn expression [34, 37], and two other SNPs in this region, rs356165 and rs356219, have been associated with PD in different cohorts $[38,39]$. Our results confirmed a new miRNA, miR-486-3p, that targeted and regulated SIRT2, and the SNP rs2241703 is located in the core miR-486-3p-binding sequence of SIRT2. The luciferase reporter assay further confirmed that this mutation significantly influenced binding affinity, suggesting that the rs2241703 SNP influences SIRT2 levels by affecting the binding efficiency of miR-486-3p to SIRT2 due to mutation from the G allele to the A allele, resulting in weakening of the inhibitory effect and high levels of SIRT2, which may contribute to a high risk of PD or accelerate the development of this disease. Furthermore, SIRT2 increased with aging $[17,28,40]$, which may explain why AA+GA genotype or A allele 


\section{Cellular Physiology Cell Physiol Biochem 2018;51:2732-2745

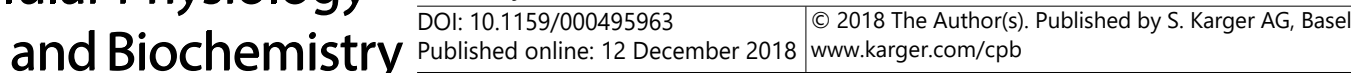 \\ Wang et al.: The Mechanism of rs2241703 Associated with Risk of PD}

carriers of rs2241703 showed a higher risk of LOPD than PD. However, more evidence is needed be verify this speculation.

Our case-control study showed that individuals in the southern Chinese Han population carrying the AA+GA genotype or A allele of rs2241703 had a higher risk of PD/LOPD. The rs2241703 SNP has not previously been associated with this disease, and only one reported case-control study of colorectal cancer in a Chinese Han population has highlighted the rs2241703 gene polymorphism, showing a minor allele frequency (MAF) $>0.05$ [41], which was consistent with our sequencing results. Notably, based on current data from the 1000 Genomes Project, we found that the rs2241703 gene polymorphism exists only in a portion of the Asian population (Chinese, Japanese, and Vietnam), showing MAF $>0.05$, which is one of the reasons why GWASs conducted in Caucasian populations have not identified this SNP as being associated with a risk of PD [42]. Furthermore, as a large number of SNPs are simultaneously examined in GWAS, an extremely low P-value threshold may be employed for genome-wide significance, potentially leading to the omission of some marginally associated genes $[43,44]$. However, considering that PD is a complex result of interactions between multiple genes and environmental factors and that the genetic backgrounds of the studied populations differ substantially, ethnicity-specific effects should be considered [45, 46]. Nevertheless, the present results suggested that the rs2241703 SNP may be a major or even the unique locus associated with PD in Chinese populations. Further studies involving a larger sample size of PD patients from different ethnic origins are needed to confirm these conclusions.

Some limitations of the present study should be acknowledged. We confirmed that SIRT2 is the target of miR-486-3p, and the rs2241703 SNP influences the binding efficiency between these molecules in vitro. Nevertheless, based on homology comparisons, the sequence in the miR-486-3p binding site of SIRT2 is not the same between humans and mice, and we therefore cannot further verify our conclusions in mice. However, we provide indirect evidence from human PBMCs to corroborate the relationship between SIRT2 and miR-486-3P. Furthermore, our aim in this study was to evaluate the rescue effect of miR-486$3 \mathrm{P}$ on the pathological process of PD, so miR-486-3P-mediated inhibition of the pathological process of PD was not addressed. In addition, although we recruited clinical samples over a significant length of time, the clinical sample size of PD patients was still not sufficiently large, and further replication studies in larger, independent populations are necessary to corroborate these results.

\section{Conclusion}

In summary, we reported for the first time a new risk-conferring polymorphism in the SIRT2 gene and explored a biological mechanism for this functional polymorphism. Through a hospital-based case-control study, we identified a significant association of the rs2241703 polymorphism located in the 3' UTR of the SIRT2 gene with PD in a Chinese Han population, and we provide further evidence showing that this SNP alters the binding efficiency of miR486-3p to SIRT2, thereby affecting the expression level of SIRT2. In turn, miR-486-3p was shown to influence neurotoxicity through SIRT2 in a PD cell model and may ultimately contribute to the progression and risk of PD. In further studies, we will explore whether miR-486-3p and SIRT2 may be useful in the diagnosis of PD and therapeutic interventions for this disease. 


\section{Cellular Physiology Cell Physiol Biochem 2018;51:2732-2745

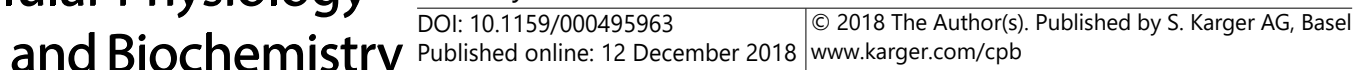

\section{Acknowledgements}

This work was supported by funding from the National Nature Science Foundation of China (81401061 and 81671181) and Guangdong Province Universities and Colleges Pearl River Scholar Funded Scheme (2017).

All experiments involving human subjects were conducted according to the principles in the Declaration of Helsinki. Written informed consent was obtained from all enrolled participants. The present study was approved by the Ethics Committee of the Affiliated Hospital of Guangdong Medical University.

\section{Disclosure Statement}

The authors declare that there is no conflict of interest.

\section{References}

1 Bendor JT, Logan TP, Edwards RH: The function of alpha-synuclein. Neuron 2013;79:1044-1066.

-2 Goedert M: NEURODEGENERATION. Alzheimer's and Parkinson's diseases: The prion concept in relation to assembled Abeta, tau, and alpha-synuclein. Science (New York, NY) 2015;349:1255555.

3 Ioannidis JP, Thomas G, Daly MJ: Validating, augmenting and refining genome-wide association signals. Nature reviews Genetics 2009;10:318-329.

4 Manolio TA, Brooks LD, Collins FS: A HapMap harvest of insights into the genetics of common disease. The Journal of clinical investigation 2008;118:1590-1605.

-5 Nalls MA, Pankratz N, Lill CM, Do CB, Hernandez DG, Saad M, DeStefano AL, Kara E, Bras J, Sharma M, Schulte C, Keller MF, Arepalli S, Letson C, Edsall C, Stefansson H, Liu X, Pliner H, Lee JH, Cheng R et al.: Large-scale meta-analysis of genome-wide association data identifies six new risk loci for Parkinson's disease. Nature genetics 2014;46:989-993.

6 Liu X, Cheng R, Verbitsky M, Kisselev S, Browne A, Mejia-Sanatana H, Louis ED, Cote LJ, Andrews H, Waters C, Ford B, Frucht S, Fahn S, Marder K, Clark LN, Lee JH: Genome-wide association study identifies candidate genes for Parkinson's disease in an Ashkenazi Jewish population. BMC medical genetics 2011;12:104.

7 Satake W, Nakabayashi Y, Mizuta I, Hirota Y, Ito C, Kubo M, Kawaguchi T, Tsunoda T, Watanabe M, Takeda A, Tomiyama H, Nakashima K, Hasegawa K, Obata F, Yoshikawa T, Kawakami H, Sakoda S, Yamamoto M, Hattori N, Murata M et al.: Genome-wide association study identifies common variants at four loci as genetic risk factors for Parkinson's disease. Nature genetics 2009;41:1303-1307.

-8 Nalls MA, Plagnol V, Hernandez DG, Sharma M, Sheerin UM, Saad M, Simon-Sanchez J, Schulte C, Lesage S, Sveinbjornsdottir S, Stefansson K, Martinez M, Hardy J, Heutink P, Brice A, Gasser T, Singleton AB, Wood NW: Imputation of sequence variants for identification of genetic risks for Parkinson's disease: a metaanalysis of genome-wide association studies. Lancet (London, England) 2011;377:641-649.

-9 Singleton A, Hardy J: The Evolution of Genetics: Alzheimer's and Parkinson's Diseases. Neuron 2016;90:1154-1163.

10 Rothgiesser KM, Erener S, Waibel S, Luscher B, Hottiger MO: SIRT2 regulates NF-kappaB dependent gene expression through deacetylation of p65 Lys310. Journal of cell science 2010;123:4251-4258.

11 Jiang W, Wang S, Xiao M, Lin Y, Zhou L, Lei Q Xiong Y, Guan KL, Zhao S: Acetylation regulates gluconeogenesis by promoting PEPCK1 degradation via recruiting the UBR5 ubiquitin ligase. Molecular cell 2011;43:33-44.

12 Serrano L, Martinez-Redondo P, Marazuela-Duque A, Vazquez BN, Dooley SJ, Voigt P, Beck DB, KaneGoldsmith N, Tong Q, Rabanal RM, Fondevila D, Munoz P, Kruger M, Tischfield JA, Vaquero A: The tumor suppressor SirT2 regulates cell cycle progression and genome stability by modulating the mitotic deposition of H4K20 methylation. Genes \& development 2013;27:639-653.

13 Donmez G, Outeiro TF: SIRT1 and SIRT2: emerging targets in neurodegeneration. EMBO molecular medicine 2013;5:344-352. 


\section{Cellular Physiology Cell Physiol Biochem 2018;51:2732-2745 \begin{tabular}{l|l|l|l|l} 
DOI: 10.1159/000495963 & 2018 The Author(s). Published by S. Karger AG, Basel \\
and Biochemistry
\end{tabular}

14 Wang F, Tong Q: SIRT2 Suppresses Adipocyte Differentiation by Deacetylating FOXO1 and Enhancing FOXO1's Repressive Interaction with PPAR\%. Molecular biology of the cell 2009;20:801-808.

15 He FF, You RY, Ye C, Lei CT, Tang H, Su H, Zhang C: Inhibition of SIRT2 Alleviates Fibroblast Activation and Renal Tubulointerstitial Fibrosis via MDM2. Cellular physiology and biochemistry : international journal of experimental cellular physiology, biochemistry, and pharmacology 2018;46:451-460.

16 Maxwell MM, Tomkinson EM, Nobles J, Wizeman JW, Amore AM, Quinti L, Chopra V, Hersch SM, Kazantsev AG: The Sirtuin 2 microtubule deacetylase is an abundant neuronal protein that accumulates in the aging CNS. Human molecular genetics 2011;20:3986-3996.

17 Wang X, Guan Q, Wang M, Yang L, Bai J, Yan Z, Zhang Y, Liu Z: Aging-related rotenone-induced neurochemical and behavioral deficits: role of SIRT2 and redox imbalance, and neuroprotection by AK-7. Drug design, development and therapy 2015;9:2553-2563.

18 Liu L, Arun A, Ellis L, Peritore C, Donmez G: SIRT2 enhances 1-methyl-4-phenyl-1, 2,3, 6-tetrahydropyridine (MPTP)-induced nigrostriatal damage via apoptotic pathway. Frontiers in aging neuroscience 2014;6:184.

19 Outeiro TF, Kontopoulos E, Altmann SM, Kufareva I, Strathearn KE, Amore AM, Volk CB, Maxwell MM, Rochet JC, McLean PJ, Young AB, Abagyan R, Feany MB, Hyman BT, Kazantsev AG: Sirtuin 2 inhibitors rescue alpha-synuclein-mediated toxicity in models of Parkinson's disease. Science (New York, NY) 2007;317:516519.

20 de Oliveira RM, Sarkander J, Kazantsev AG, Outeiro TF: SIRT2 as a Therapeutic Target for Age-Related Disorders. Frontiers in pharmacology 2012;3:82.

-21 de Oliveira RM, Vicente Miranda H, Francelle L, Pinho R, Szego EM, Martinho R, Munari F, Lazaro DF, Moniot S, Guerreiro P, Fonseca-Ornelas L, Marijanovic Z, Antas P, Gerhardt E, Enguita FJ, Fauvet B, Penque D, Pais TF, Tong Q, Becker S et al.: The mechanism of sirtuin 2-mediated exacerbation of alpha-synuclein toxicity in models of Parkinson disease. PLoS Biol 2017;15:e2000374.

22 Guerreiro PS, Huang Y, Gysbers A, Cheng D, Gai WP, Outeiro TF, Halliday GM: LRRK2 interactions with $\alpha$-synuclein in Parkinson's disease brains and in cell models. J Mol Med (Berl) 2013;91:513-522.

-23 Lemos V, de Oliveira RM, Naia L, Szego E, Ramos E, Pinho S, Magro F, Cavadas C, Rego AC, Costa V, Outeiro TF, Gomes P: The NAD+-dependent deacetylase SIRT2 attenuates oxidative stress and mitochondrial dysfunction and improves insulin sensitivity in hepatocytes. Human molecular genetics 2017;26:41054117.

24 Sayd S, Junier MP, Chneiweiss H: SIRT2, a multi-talented deacetylase. Med Sci (Paris) 2014;30:532-536.

25 Tang X, Chen XF, Wang NY, Wang XM, Liang ST, Zheng W, Lu YB, Zhao X, Hao DL, Zhang ZQ, Zou MH, Liu DP, Chen HZ: SIRT2 Acts as a Cardioprotective Deacetylase in Pathological Cardiac Hypertrophy. Circulation 2017;136:2051-2067.

26 Zhou W, Ni TK, Wronski A, Glass B, Skibinski A, Beck A, Kuperwasser C: The SIRT2 Deacetylase Stabilizes Slug to Control Malignancy of Basal-like Breast Cancer. Cell reports 2016;17:1302-1317.

27 North BJ, Rosenberg MA, Jeganathan KB, Hafner AV, Michan S, Dai J, Baker DJ, Cen Y, Wu LE, Sauve AA, van Deursen JM, Rosenzweig A, Sinclair DA: SIRT2 induces the checkpoint kinase BubR1 to increase lifespan. The EMBO journal 2014;33:1438-1453.

28 Fourcade S, Outeiro TF, Pujol A: SIRT2 in age-related neurodegenerative disorders. Aging 2018;10:295-296.

-29 Singh P, Hanson PS, Morris CM: Sirtuin 2 Oxidative Stress and Is Elevated in Neurodegeneration. Parkinsons Dis 2017;2017: 2643587.

30 Wang X, Li W, Ma L, Gao J, Liu J, Ping F, Nie M: Association study of the miRNA-binding site polymorphisms of CDKN2A/B genes with gestational diabetes mellitus susceptibility. Acta diabetologica 2015;52:951-958.

-31 Preskill C, Weidhaas JB: SNPs in microRNA binding sites as prognostic and predictive cancer biomarkers. Critical reviews in oncogenesis 2013;18:327-340.

-32 Xu J, Tian S, Yin Z, Wu S, Liu L, Qian Y, Pei D, Gao W, Xu J, Yin Y, Liu P, Shu Y: MicroRNA-binding site SNPs in deregulated genes are associated with clinical outcome of non-small cell lung cancer. Lung cancer (Amsterdam, Netherlands) 2014;85:442-448.

33 Ghanbari M, Darweesh SK, de Looper HW, van Luijn MM, Hofman A, Ikram MA, Franco OH, Erkeland SJ, Dehghan A: Genetic Variants in MicroRNAs and Their Binding Sites Are Associated with the Risk of Parkinson Disease. Human mutation 2016;37:292-300.

34 Sotiriou S, Gibney G, Baxevanis AD, Nussbaum RL: A single nucleotide polymorphism in the 3'UTR of 


\section{Cellular Physiology Cell Physiol Biochem 2018;51:2732-2745

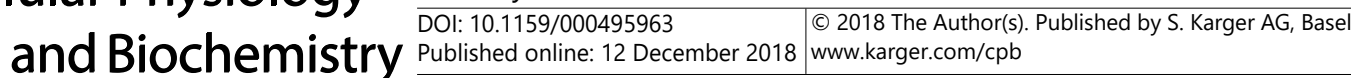

Wang et al.: The Mechanism of rs2241703 Associated with Risk of PD

the SNCA gene encoding alpha-synuclein is a new potential susceptibility locus for Parkinson disease. Neuroscience letters 2009;461:196-201.

-35 Wu Z, Wang P, Song C, Wang K, Yan R, Li J, Dai L: Evaluation of miRNA-binding-site SNPs of MRE11A, NBS1, RAD51 and RAD52 involved in HRR pathway genes and risk of breast cancer in China. Mol Genet Genomics 2015;290:1141-1153.

-36 Ma L, Xu M, Li D, Han Y, Wang Z, Yuan H, Ma J, Zhang W, Jiang H, Pan Y, Wang L: A miRNA-binding-site SNP of MSX1 is Associated with NSOC Susceptibility. Journal of dental research 2014;93:559-564.

-37 Kabaria S, Choi DC, Chaudhuri AD, Mouradian MM, Junn E: Inhibition of miR-34b and miR-34c enhances alpha-synuclein expression in Parkinson's disease. FEBS letters 2015;589:319-325.

38 Pan F, Dong H, Ding H, Ye M, Liu W, Wu Y, Zhang X, Chen Z, Luo Y, Ding X: SNP rs356219 of the alphasynuclein (SNCA) gene is associated with Parkinson's disease in a Chinese Han population. Parkinsonism \& related disorders 2012;18:632-634.

39 Cardo LF, Coto E, de Mena L, Ribacoba R, Lorenzo-Betancor O, Pastor P, Samaranch L, Mata IF, Diaz M, Moris G, Menendez M, Corao AI, Alvarez V: A search for SNCA 3' UTR variants identified SNP rs356165 as a determinant of disease risk and onset age in Parkinson's disease. Journal of molecular neuroscience : MN 2012;47:425-430.

40 Park SH, Zhu Y, Ozden O, Kim HS, Jiang H, Deng CX, Gius D, Vassilopoulos A: SIRT2 is a tumor suppressor that connects aging, acetylome, cell cycle signaling, and carcinogenesis. Translational cancer research 2012;1:15-21.

-41 Yang Y, Ding J, Gao Z-G, Wang Z-J: A variant in SIRT2 gene 3' -UTR is associated with susceptibility to colorectal cancer. Oncotarget 2017;8:41021-41025.

42 Verstraeten A, Theuns J, Van Broeckhoven C: Progress in unraveling the genetic etiology of Parkinson disease in a genomic era. Trends Genet 2015;31:140-149.

-43 Dong J, Hu Z, Wu C, Guo H, Zhou B, Lv J, Lu D, Chen K, Shi Y, Chu M, Wang C, Zhang R, Dai J, Jiang Y, Cao S, Qin Z, Yu D, Ma H, Jin G, Gong J et al.: Association analyses identify multiple new lung cancer susceptibility loci and their interactions with smoking in the Chinese population. Nature genetics 2012;44:895-899.

44 Panagiotou OA, Ioannidis JP: What should the genome-wide significance threshold be? Empirical replication of borderline genetic associations. International journal of epidemiology 2012;41:273-286.

45 Peeraully T, Tan EK: Genetic variants in sporadic Parkinson's disease: East vs West. Parkinsonism Relat Disord 2012;18 Suppl 1:S63-65.

46 Chang XL, Mao XY, Li HH, Zhang JH, Li NN, Burgunder JM, Peng R, Tan EK: Association of GWAS loci with PD in China. Am J Med Genet B Neuropsychiatr Genet 2011;156b:334-339. 